\title{
Study for System of Nonlinear Differential Equations with Riemann-Liouville Fractional Derivative
}

\author{
Yanping Zheng*, Wenxia Wang \\ Department of Mathrmatics, Taiyuan Normal University, Taiyuan, China \\ Email: *zhengyanping2003@126.com
}

Received April 19, 2013; revised May 19, 2013; accepted May 29, 2013

Copyright (C) 2013 Yanping Zheng, Wenxia Wang. This is an open access article distributed under the Creative Commons Attribution License, which permits unrestricted use, distribution, and reproduction in any medium, provided the original work is properly cited.

\begin{abstract}
In this work, we study existence theorem of the initial value problem for the system of fractional differential equations $D^{\alpha} \bar{x}(t)=A \bar{x}(t),\left.t^{1-\alpha} \bar{x}(t)\right|_{x=0}=\bar{b}$, where $D^{\alpha}$ denotes standard Riemann-Liouville fractional derivative, $0<\alpha<1$, $\bar{x}(t)=\left[x_{1}(t), x_{2}(t), \cdots, x_{n}(t)\right]^{\mathrm{T}}, \bar{b}=\left[b_{1}, b_{2}, \cdots, b_{n}\right]^{\mathrm{T}}$ and $A$ is a square matrix. At the same time, power-type estimate for them has been given.

Keywords: Riemann-Liouville Fractional Derivative; Weighted Cauchy-Type Problem; Fractional Differential Equations
\end{abstract}

\section{Introduction}

Let $M_{n}$ denote the $n \times n$ matrix over real fields $R$ or complex fields $C$. For $h>0$,

$$
\begin{aligned}
& C_{r}^{0}([0, h]):= \\
& \left\{f \in C^{0}((0, h]): \lim _{t \rightarrow 0^{+}} t^{r} f(t) \text { exists and is finite }\right\},
\end{aligned}
$$

here $C^{0}((0, h])$ is the usual space of continuous functions on $(0, h]$, which is a Banach space with the norm

$$
\|f\|_{r}:=\max _{0 \leq t \leq h} t^{r}|f(t)| \text {. }
$$

The space $C_{1-\alpha}^{\alpha}([0, h])$ is defined by $C_{1-\alpha}^{\alpha}([0, h]):=\left\{f \in C_{1-\alpha}([0, h]):\right.$ there exists $c \in R$ and $f^{*} \in C_{1-\alpha}^{0}([0, h])$ s.t. $\left.f(t)=c t^{\alpha-1}+I^{\alpha} f^{*}(t)\right\}$.

(see [1]).

The existence of solution of initial value problems for fractional order differential equations have been studied in many literatures such as [1-4]. In this paper, we present the analysis of the system of fractional differential equations

$$
\left\{\begin{array}{l}
D^{\alpha} \bar{x}(t)=A \bar{x}(t), \\
\left.t^{1-\alpha} \bar{x}(t)\right|_{t=0}=\bar{b}
\end{array}\right.
$$

*Corresponding author. where $D^{\alpha}$ denotes standard Riemann-Liouville fractional derivative, where

$$
\begin{aligned}
& \bar{x}(t)=\left[x_{1}(t), x_{2}(t), \cdots, x_{n}(t)\right]^{\mathrm{T}}, \\
& D^{\alpha} \bar{x}(t)=\left[D^{\alpha} x_{1}(t), D^{\alpha} x_{2}(t), \cdots, D^{\alpha} x_{n}(t)\right]^{\mathrm{T}}, \\
& 1 / 2<\alpha<1,
\end{aligned}
$$

$\bar{b}=\left[b_{1}, b_{2}, \cdots, b_{n}\right]^{\mathrm{T}}$ and $A$ is a square.

To prove the main result, we begin with some definitions and lemmas. For details, see [1-5].

Definition 1.1 Let $f$ be a continuous function defined on $[a, b]$ and $n-1 \leq \alpha<n, n \in N$. Then the expression

$$
D_{a+}^{\alpha} f(x)=\frac{1}{\Gamma(n-\alpha)} \frac{\mathrm{d}^{n}}{\mathrm{~d} x^{n}} \int_{a}^{x} \frac{f(t)}{(x-t)^{\alpha-n+1}} \mathrm{~d} t, x>a
$$

is called left-sided fractional derivatives of order $\alpha$.

Definition 1.2 Let $f$ be a continuous function defined on $[a, b]$ and $\alpha>0$. Then the expression

$$
I_{a+}^{\alpha} f(x)=\frac{1}{\Gamma(\alpha)} \int_{a}^{x} \frac{f(t)}{(x-t)^{-\alpha+1}} \mathrm{~d} t, x>a
$$

is called left-sided fractional integral of order $\alpha$.

Lemma 1.3 Given $A \in M_{n}$ with eigenvalues $\lambda_{1}, \lambda_{2}, \cdots, \lambda_{n}$ in any prescribed order, there is a unitary 
matrix $U \in M_{n}$ such that $U^{*} A U=T=\left[t_{i j}\right]$ is upper triangular with diagonal entries $t_{i i}=\lambda_{i}, \quad i=1, \cdots, n$. That is, every square matrix $A$ is unitarily equivalent to triangular matrix whose entries are the eigenvalues of $A$ in a prescribed order. Further more, if $A \in M_{n}(R)$ and if all the eigenvalues of $A$ are real, then $U$ may be chosen to be real and orthogonal.

Lemma 1.4 Assume that $f \in C^{0}\left(R_{0}^{+}\right) \cap \operatorname{Loc}^{1}\left(R_{0}^{+}\right)$ with fractional derivative of order $0<\alpha<1$ that belongs to $C^{0}\left(R_{0}^{+}\right) \cap \operatorname{Loc}^{1}\left(R_{0}^{+}\right)$. Then

$$
I^{\alpha} D^{\alpha} f(x)=f(x)+C x^{\alpha-1}
$$

for some $c \in R$. When the function $f \in C^{0}\left(R^{+}\right)$, then $c=0$, where

$$
R_{0}^{+}=\{x \in R, x>0\} \text { and } R^{+}=\{x \in R, x \geq 0\} .
$$

Lemma 1.5 (Schauder's fixed theorem) Assume $\Omega$ is a relative subset of a convex set $K$ in a normed space $X$. Let $A: \bar{\Omega} \rightarrow K$ be a compact map with $0 \in \Omega$. Then either

(A $\left.\mathrm{A}_{1}\right) A$ has a fixed point in $\bar{\Omega}$, or

$\left(\mathrm{A}_{2}\right)$ there is a $x \in \partial \Omega$ and a $\lambda<1$ such that $x=\lambda A x$.

Now, let's us give some hypotheses:

H1: $f(t, x)$ is continuous on $R^{+} \times R$ and is such that

$$
|f(t, x)| \leq t^{\mu} \varphi(t) \mathrm{e}^{-\sigma t}|x|^{m}, \mu \geq 0, m>1, \sigma>0,
$$

where $\varphi(t)$ is a continuous function on $R^{+}$.

H2: $f(t, x)$ is continuous on $R^{+} \times R$ and is such that

$$
|f(t, x)| \leq t^{\mu} \varphi(t)|x|^{m}, \mu \geq 0, m>1,
$$

where $\varphi(t)$ is a continuous function on $R^{+}$.

Lemma 1.6 Let $1 / 2<\alpha<1$. If we assume that $0<q<1 / 1-\alpha$, then the initial value problem

$$
\left\{\begin{array}{l}
D^{\alpha} x(t)=x^{q}(t)+y(t), \\
\left.t^{1-\alpha} x(t)\right|_{t=0}=b,
\end{array}\right.
$$

where

$$
\begin{gathered}
y(t) \in C_{1-\alpha}^{0}([0, h]) \cap L^{1}((0, h)), \\
x^{q}(t) \in C_{1-\alpha}^{0}([0, h]) \cap L^{1}((0, h)),
\end{gathered}
$$

has at least a solution $x(t) \in C_{1-\alpha}^{0}([0, h]) \cap L^{1}((0, h))$ for $h>0$ sufficiently small.

\section{Proof. If}

$$
x^{q}(t) \in C_{1-\alpha}^{0}([0, h]) \cap L^{1}((0, h)),
$$

then $q(\alpha-1)>-1$, by Lemma 1.4 , We are therefore reduced the initial problem to the nonlinear integral equation

$$
\begin{aligned}
& x(t)=b t^{\alpha-1} \\
& +\frac{1}{\Gamma(\alpha)}\left(\int_{0}^{t}(t-s)^{\alpha-1} x^{q}(s) \mathrm{d} s+\int_{0}^{t}(t-s)^{\alpha-1} y(s) \mathrm{d} s\right) .
\end{aligned}
$$

The existence of a solution to Problem (3) can be formulated as a fixed point equation $T x=x$, where

$$
\begin{aligned}
& (T x)(t)=b t^{\alpha-1} \\
& +\frac{1}{\Gamma(\alpha)}\left(\int_{0}^{t}(t-s)^{\alpha-1} x^{q}(s) \mathrm{d} s+\int_{0}^{t}(t-s)^{\alpha-1} y(s) \mathrm{d} s\right)
\end{aligned}
$$

in the space $C_{1-\alpha}^{0}([0, h]) \cap L^{1}((0, h))$.

Define

$$
\begin{aligned}
& S=\left\{x \in C_{1-\alpha}^{0}([0, h]):\left\|x-b t^{\alpha-1}\right\|_{1-\alpha}\right. \\
& \left.\leq r+\frac{1}{\alpha} h^{2 \alpha-1}\|y\|_{1-\alpha}\right\} .
\end{aligned}
$$

Clearly, it is closed, convex and nonempty.

Step I. We shall prove that we note that $T S \subseteq S$.

We note that

$$
\begin{aligned}
& \left\|T x-b t^{\alpha-1}\right\|_{1-\alpha}=\max _{t \in[0, h]} \frac{t^{1-\alpha}}{\Gamma(\alpha)} \\
& \cdot\left\{\left|\int_{0}^{t}(t-s)^{\alpha-1} x^{q}(s) \mathrm{d} s+\int_{0}^{t}(t-s)^{\alpha-1} y(s) \mathrm{d} s\right|\right\} \\
& \leq \max _{t \in[0, h]} \frac{t^{1-\alpha}}{\Gamma(\alpha)}\left\{\left|\int_{0}^{t}(t-s)^{\alpha-1} s^{q(\alpha-1)} s^{q(1-\alpha)} x^{q}(s) \mathrm{d} s\right|\right. \\
& \left.\quad+\left|\int_{0}^{t}(t-s)^{\alpha-1} s^{\alpha-1} s^{1-\alpha} y(s) \mathrm{d} s\right|\right\} \\
& =\max _{t \in[0, h]} \frac{t^{1-\alpha}}{\Gamma(\alpha)}\left\{\left|\int_{0}^{t}(t-s)^{\alpha-1} s^{q(\alpha-1)} s^{q(1-\alpha)} x^{q}(s) \mathrm{d} s\right|\right\} \\
& \left.\quad+\max _{t \in[0, h]} \frac{t^{1-\alpha}}{\Gamma(\alpha)}\right) \mid\left\{\int_{0}^{t}(t-s)^{\alpha-1} s^{\alpha-1} s^{1-\alpha} y(s) \mathrm{d} s \mid\right\} \\
& \leq \frac{\Gamma(q(\alpha-1)+1)}{\Gamma(q(\alpha-1)+1+\alpha) \Gamma(\alpha)} h^{q(\alpha-1)+1}\|x\|_{1-\alpha}^{q}+\frac{1}{\alpha} h^{2 \alpha-1}\|y\|_{1-\alpha} .
\end{aligned}
$$

Since $\|x\|_{1-\alpha} \leq r+|b|+\frac{1}{\alpha} h^{2 \alpha-1}\|y\|_{1-\alpha}$, it will be sufficient to impose

$$
\begin{aligned}
& \left\|x-b t^{\alpha-1}\right\|_{1-\alpha} \\
& \leq \text { const. } h^{q(\alpha-1)+1}\left(r+|b|+\frac{1}{\alpha} h^{2 \alpha-1}\|y\|_{1-\alpha}\right)^{q} \leq r .
\end{aligned}
$$

In view of the assumption $q(\alpha-1)+1>0$, the second estimate is satisfied if say $r=|b|$ and $h$ is chosen sufficiently small.

Step II. We shall prove that the operator $T$ is compact. To prove the compactness of 


$$
T: C_{1-\alpha}^{0}([0, h]) \rightarrow C_{1-\alpha}^{0}([0, h])
$$

defined by (5), it will be sufficient to argue on the operator

$$
T_{*}: C^{0}([0, h]) \rightarrow C^{0}([0, h])
$$

defined in this way:

$$
\left(T_{*} x\right)(t)=t^{1-\alpha} T\left(t^{\alpha-1} x(t)\right) .
$$

We have $T_{*} x=b+T^{*} x$ where the operator

$$
\begin{aligned}
\left(T^{*} x\right)(t) & =\frac{t^{1-\alpha}}{\Gamma(\alpha)}\left(\int_{0}^{t}(t-s)^{\alpha-1} s^{q(\alpha-1)} x^{q}(s) \mathrm{d} s\right. \\
& \left.+\int_{0}^{t}(t-s)^{\alpha-1} s^{\alpha-1} y(s) \mathrm{d} s\right) .
\end{aligned}
$$

Turn out to be compact from classical sufficient conditions, since $q(\alpha-1)>-1, \alpha-1>-1$. By Lemma 1.5, we have that Problem (3) has least a solution.

The proof is complete.

Lemma 1.7 Suppose that $f(t, x)$ satisfies $\mathrm{H} 1$, $\mu-(m-1)(1-\alpha)>0$ and $\alpha>1 / 2$. If $\|\varphi\|_{q}<L$ for some $q>1 / \alpha$, then the problem

$$
\left\{\begin{array}{l}
D^{\alpha} x(t)=f(t, x), \\
\left.t^{1-\alpha} x(t)\right|_{t=0}=b,
\end{array}\right.
$$

exists a positive constant $C$ such that $|x(t)| \leq C t^{\alpha-1}$, $t>0$.

Lemma 1.8 Let $x \in C_{1-\alpha}^{0}([0, h])$ with $\alpha>1 / 2$. Suppose further that $\mu-(m-1)(1-\alpha)>0$. Then Problem (6) and its associated integral equation

$$
x(t)=b t^{\alpha-1}+\frac{1}{\Gamma(\alpha)} \int_{0}^{t}(t-s)^{\alpha-1} f(s, x(s)) \mathrm{d} s
$$

are equivalent.

Lemma 1.9 Assume that $\alpha>1 / 2 . \quad f(t, x)$ satisfies H2, and $\|\varphi\|_{q}<K$ for some $q>1 / 2$. Suppose further that $\mu+1 / p<m(1-\alpha)$, then there exists $C>0$ and $0<\delta<1-\alpha$ such that any solution of (6) exists globally and satisfies

$$
|x(t)| \leq C t^{-\delta}, t \geq a>0 .
$$

\section{Main Results}

Theorem 2.1 Let $A \in M_{n}$ then initial problem (*) has a solution $\bar{x}(t) \in R^{n}$, where

$$
\begin{gathered}
\bar{x}(t)=\left[x_{1}(t), x_{2}(t), \cdots, x_{n}(t)\right]^{\mathrm{T}}, \\
x_{i}(t) \in C_{1-\alpha}^{0}([0, h]) \cap L^{1}((0, h))
\end{gathered}
$$

for all $i=1,2, \cdots, n$ and sufficiently small $h>0$.

Proof. Given $A \in M_{n}$ with eigenvalues $\lambda_{1}, \lambda_{2}, \cdots, \lambda_{n}$ by Lemma 1.3 , there is a unitary matrix $U \in M_{n}$ such that

$$
U^{*} A U=T=\left[t_{i j}\right]
$$

is upper triangular with diagonal entries $t_{i i}=\lambda_{i}, i=1, \cdots, n$. Let $\bar{y}(t)=U^{*} \bar{x}(t)$, we have

$$
\begin{aligned}
D^{\alpha} \bar{y}(t) & =U^{*} D^{\alpha} \bar{x}(t)=U^{*} A \bar{x}(t) \\
& =U^{*} A U \bar{y}(t)=T \bar{y}(t) .
\end{aligned}
$$

At the same time, the initial problem $(*)$ changed into

$$
\left\{\begin{array}{l}
D^{\alpha} \bar{y}(t)=T \bar{y}(t), \\
\left.t^{1-\alpha} \bar{y}(t)\right|_{t=0}=U^{*} \bar{b}
\end{array}\right.
$$

Now, let's consider the problem $(* *)$.

Clearly, the problem $(* *)$ is equivalent to the following $n$ problems

$$
\left\{\begin{array}{l}
D^{\alpha} y_{i}(t)=\sum_{j=i}^{n} t_{i j} y_{j}(t), \\
\left.t^{1-\alpha} y_{i}(t)\right|_{t=0}=b_{i},
\end{array}\right.
$$

for $i, j=1,2, \cdots, n$. where $b_{i}$ is the $i$ th entries of the vector $U^{*} \bar{b}$.

Consider the weighed Cauchy-type problem

$$
\left\{\begin{array}{l}
D^{\alpha} y_{n}(t)=t_{n n} y_{n}(t), \\
\left.t^{1-\alpha} y_{n}(t)\right|_{t=0}=b_{n} .
\end{array}\right.
$$

In Lemma 1.6, take $q=1, y(t)=0$. Then by lemma 1.6, $\exists h>0$, s.t. the above problem has at least a solution

$$
y_{n}(t) \in C_{1-\alpha}^{0}([0, h]) \cap L^{1}((0, h)) .
$$

Consider the following weighed Cauchy-type problem

$$
\left\{\begin{array}{l}
D^{\alpha} y_{n-1}(t)=t_{n-1, n-1} y_{n-1}(t)+t_{n-1, n} y_{n}(t), \\
\left.t^{1-\alpha} y_{n-1}(t)\right|_{t=0}=b_{n-1} .
\end{array}\right.
$$

In Lemma 1.6, take $q=1, y(t)=t_{n-1, n} y_{n}(t)$. Then by Lemma 1.6, $\exists h>0$, s.t. the above problem has at least a solution $y_{n}(t) \in C_{1-\alpha}^{0}([0, h]) \cap L^{1}((0, h))$.

Similarly, there has at least a solution in

$$
C_{1-\alpha}^{0}([0, h]) \cap L^{1}((0, h))
$$

for the rest $n$-2 initial problem in $(* *)$, denote by $y_{n-2}(t), y_{n-3}(t), \cdots, y_{1}(t)$ respectively. And therefore, there has at least a solution

$$
\bar{y}(t)=\left[y_{1}(t), y_{2}(t), \cdots, y_{n}(t)\right]^{\mathrm{T}}
$$

of the problem $\left(^{* *}\right)$. Let $\bar{x}(t)=U \bar{y}(t)$, it is required for us.

The proof is completed. 
Since the problem $(* *)$ is equivalent to the following $n$ problems

$$
\left\{\begin{array}{l}
D^{\alpha} y_{i}(t)=\sum_{j=i}^{n} t_{i j} y_{j}(t), \\
\left.t^{1-\alpha} y_{i}(t)\right|_{t=0}=b_{i},
\end{array}\right.
$$

for $i, j=1,2, \cdots, n$. where $b_{i}$ is the $i$ th entries of the vector $U^{*} \bar{b}$. Next, we shall discuss these equations in (9).

Theorem 2.2 Assume that the right hand of these equations in (9) satisfied $\mathrm{H} 1, \mu-(m-1)(1-\alpha)>0$, $\alpha>1 / 2$ and $\|\varphi\|_{q}<L$ for some $q>1 / \alpha$, If the solution of the problems $(* *)$ denoted by

$$
\bar{x}(t)=\left[x_{1}(t), x_{2}(t), \cdots, x_{n}(t)\right]^{\mathrm{T}},
$$

then there exists some constant $C>0$ such that $\left|x_{i}(t)\right| \leq\|U\|_{\infty} C t^{\alpha-1}, t>0$ for all $i=1,2, \cdots, n$.

Proof. Similar to the proof of Theorem 2.1, now consider the following weighted Cauchy-type problem

$$
\left\{\begin{array}{l}
D^{\alpha} y_{n}(t)=t_{n n} y_{n}(t), \\
\left.t^{1-\alpha} y_{n}(t)\right|_{t=0}=b_{n} .
\end{array}\right.
$$

Then by Lemma 1.7, there exists some constant $C_{n}>0$ such that $\left|y_{n}(t)\right| \leq C_{n} t^{\alpha-1}, t>0$.

Consider the following problem

$$
\left\{\begin{array}{l}
D^{\alpha} y_{n-1}(t)=t_{n-1, n-1} y_{n-1}(t)+t_{n-1, n} y_{n}(t), \\
\left.t^{1-\alpha} y_{n-1}(t)\right|_{t=0}=b_{n-1} .
\end{array}\right.
$$

Then by Lemma 1.7, there exists some constant $C_{n-1}>0$ such that $\left|y_{n-1}(t)\right| \leq C_{n-1} t^{\alpha-1}, t>0$.

Similarly, there exist some positive constants $C_{n-2}, C_{n-3}, \cdots, C_{1}$ such that

$$
\left|y_{i}(t)\right| \leq C_{i} t^{\alpha-1}, t>0 .
$$

for all $i=n-2, n-3, \cdots, 1$.

Let $\bar{x}(t)=U \bar{y}(t), C=\max _{1 \leq i \leq n}\left\{C_{i}\right\}$. Then we have

$$
\left|x_{i}(t)\right| \leq\|U\|_{\infty} C t^{\alpha-1}, t>0,
$$

for all $i=1,2, \cdots, n$.

The proof is completed.
Theorem 2.3 Assume that $\alpha>1 / 2$, the right-hand of these equations in (9) satisfied $\mathrm{H} 2$, and $\|\varphi\|_{q}<K$

For some $q>1 / 2$. Suppose further that

$$
\mu+1 / p<m(1-\alpha) .
$$

If denote solution of the problems $(* *) \bar{x}(t)$ by

$$
\bar{x}(t)=\left[x_{1}(t), x_{2}(t), \cdots, x_{n}(t)\right]^{\mathrm{T}} .
$$

Then there exists some constant $C>0$ and $0<\delta<1-\alpha$, such that

$$
\left|x_{i}(t)\right| \leq\|U\|_{\infty} C t^{-\delta}, t \geq a>0,
$$

for all $i=1,2, \cdots, n$.

Using Lemmas 1.3 and 1.9, the proof is similar to Theorem 2.2. Therefore, it is omitted.

\section{Acknowledgements}

This research was supported by the NNSF of China (10961020), the Science Foundation of Qinghai Province of China (2012-Z-910) and the University Natural Science Research Develop Foundation of Shanxi Province of China (20111021).

\section{REFERENCES}

[1] K. M. Furati and N.-E. Tatar, "Power-Type Estmates for a Nonlear Fractional Differential Equation,” Journal of Nonlinear Analysis, Vol. 62, No. 6, 2005, pp. 1025-1036. doi:10.1016/j.na.2005.04.010

[2] V. Daftardar-Gejji and A. Babakhani, “Analysis of a SysTem of Fractional Differential Equations,” Journal of Mathematical Analysis and Applications, Vol. 293, No. 2, 2004, pp. 511-512. doi:10.1016/j.jmaa.2004.01.013

[3] D. Delbosco and L. Rodino, "Existence and Uniqueness for a Nonlinear Fractional Differential Equation,” Journal of Mathematical Analysis and Applications, Vol. 204, No. 2, 1996, pp. 511-512. doi:10.1006/jmaa.1996.0456

[4] I. Podlubny, "Fractional Differential Equtions," Academic Press, New York, 1999.

[5] R. A. Horn and C. R. Johnson, "Matrix Analysis,” Cambridge University press, London, 1985. 